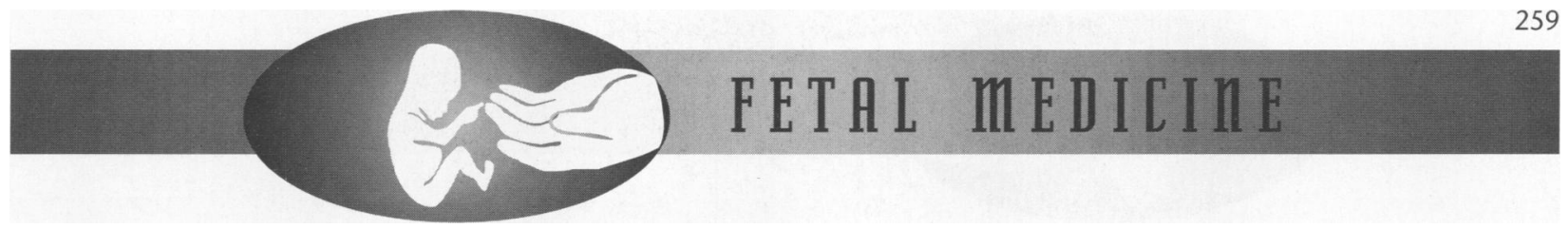

\title{
Fetal Medicine
}

As THE MYSTERIES of fetal development unfold, opportunities abound and questions proliferate. This special issue of THE WESTERN JOURNAL OF MEDICINE, with authors so capably recruited and papers so carefully edited, is the work of James Goldberg, MD, Associate Professor of Obstetrics, Gynecology and Reproductive Sciences at the University of California, San Francisco. Much of the issue addresses the question, "Can we?" From basic research and technical standpoints, the answer is "Yes," "Sometimes," "Almost," or "We hope so." From the financing standpoint, the answer is, "So far," "It depends," or "We will know when controlled studies on cost-effectiveness are conducted." Clearly, however, we have the nascent ability to diagnose and treat fetal conditions. These advances are dramatic testimony to basic, animal, and human research and touching testimony to the courage and yearnings of parents-mothers, especially, because they knowingly take risks-and of clinical scientists.

Where do we go from here? We need more science, more trials, more rigorous study of "Does it make a difference?" and "To whom?" At the same time, we and other members of society ask, "Should we?" and "Ought we?" To help answer these questions, the viewpoints of both scientists and ethicists are well represented in this special issue. As with all genuinely complex issues, discussions will be never-ending. This is a beginning.

This issue is more than a status report. It documents triumphs, asks good questions, and maps the course for future research and thinking.

LINDA HAWES CLEVER, MD

\section{Introduction}

ADVANCES IN MATERNAL FETAL MEDICINE and reproductive genetics have served to focus attention on the fetus. While we still place much concern on the condition of the pregnant woman, the effects of her condition or therapy on the fetus now are also considered. This has been an important change in outlook, brought about primarily by our improved ability to "examine" the fetus in utero, which has opened new areas of investigation in the evolving field of "fetal medicine."

Initially, this improved ability to examine the fetus made possible advances in diagnosing fetal congenital anomalies. These included the analysis of a variety of fetal tissues including amniotic fluid cells, chorionic villi, blood, liver, skin, and muscle. Another important advance was identifying fetal structural anomalies by high resolution ultrasound. These approaches allowed many couples the possibility of only having unaffected offspring. It also identified those fetuses that would need early surgical or metabolic therapy so that they might be delivered in tertiary care centers. While these advances were substantial, it was not until the advent of fetal therapy that the field of fetal medicine became firmly established.

Fetal therapy is still in its infancy. Approaches to repairing structural malformations and treating metabolic disorders are being attempted in several centers world- wide. Because of the highly experimental nature of many of these treatments, only a few centers are undertaking them. This has necessitated worldwide referrals and consultations taking place by phone, overnight courier, and the internet.

This special issue of The WeSTERn Journal of MEDICINE was organized to discuss these issues and present the readers with a status report. Investigators discuss both the current state of fetal diagnosis and innovations in fetal therapy. The other area that receives major focus is one that is vitally important in this field-the ethical and legal status of a fetus and fetal tissues. As one can readily see, we are not only dealing with the status of the developing fetus, but of the mother as well. It is evident that much serious thought has gone into examining these issues.

I wish to thank Holly Smith, MD, and Linda Clever, $\mathrm{MD}$, for giving me the opportunity to organize this special issue. I hope they ask me to do an update in ten years. I also wish to thank all of the authors for the incredible amount of work that they put into their manuscripts.

\footnotetext{
JAMES D. GOLDBERG, MD

Special Issue Editor

Associate Professor of Obstetrics, Gynecology and Reproductive Sciences

University of California, San Francisco, School of Medicine
} 